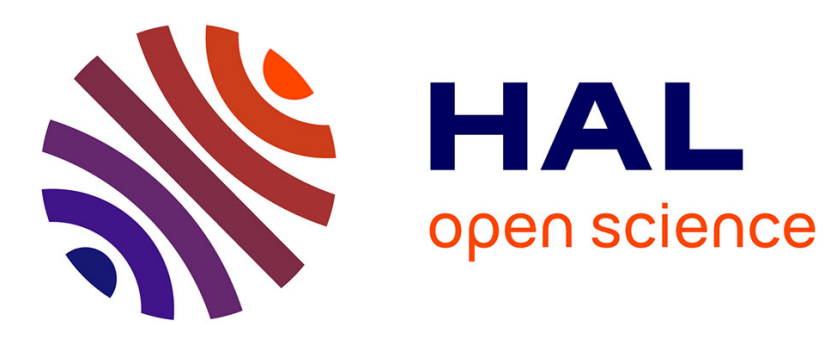

\title{
Deep rule-based classifier for finger knuckle pattern recognition system
}

\author{
Abdelouahab Attia, Zahid Akhtar, Nour Elhouda Chalabi, Sofiane Maza, \\ Nour Elhouda Chalabi, Youssef Chahir
}

\section{- To cite this version:}

Abdelouahab Attia, Zahid Akhtar, Nour Elhouda Chalabi, Sofiane Maza, Nour Elhouda Chalabi, et al.. Deep rule-based classifier for finger knuckle pattern recognition system. Evolving Systems, 2021, 1, 10.1007/s12530-020-09359-w . hal-03002570

\section{HAL Id: hal-03002570 \\ https://hal.science/hal-03002570}

Submitted on 12 Nov 2020

HAL is a multi-disciplinary open access archive for the deposit and dissemination of scientific research documents, whether they are published or not. The documents may come from teaching and research institutions in France or abroad, or from public or private research centers.
L'archive ouverte pluridisciplinaire HAL, est destinée au dépôt et à la diffusion de documents scientifiques de niveau recherche, publiés ou non, émanant des établissements d'enseignement et de recherche français ou étrangers, des laboratoires publics ou privés. 


\title{
Deep rule-based classifier for finger knuckle pattern recognition system
}

\author{
Abdelouahab Attia ${ }^{1,3} \cdot$ Zahid Akhtar $^{2} \cdot$ Nour Elhouda Chalabi $^{3} \cdot$ Sofiane Maza ${ }^{1} \cdot$ Youssef Chahir $^{4}$
}

\begin{abstract}
In this paper, we proposed a novel finger knuckle pattern (FKP) based personal authentication system using multilayer deep rule based (DRB) classifier. The presented approach is completely data-driven and fully automatic. However, the DRB classifier is generic and can be used in variety of classification or prediction problems. In particular, from the input finger knuckle, two kinds of features (i.e., Binarized Statistical Image Features and Gabor Filer bank) are extracted, which are then fed to fuzzy rules based DRB classifier to determine whether the user is genuine or impostor. Experimental results in the form of accuracy, error equal rate (EER) and receiver operating characteristic (ROC) curves demonstrate that presented DRB classifier is a powerful tool in FKP based biometric identification system. Experiments are reported using publicly available FKP PolyU database provided by University of Hong Kong. Experiments using this database show that the presented framework, in this study, can attain performance better than previously proposed methods. Moreover, score level fusion of all FKP modalities with BSIF + DRB yielded an equal error rate of $0.19 \%$ and an accuracy of $99.65 \%$.
\end{abstract}

Keywords Deep rule based classifier · BSIF · Gabor filter bank · Finger knuckle pattern

\section{Introduction}

In today's highly interconnected society, automated personal identification methods have become crucial for security and privacy (Angelov and Gu 2018; Bao et al. 2018; Angelov and Sperduti 2016). One of person recognition methods is biometrics, which is considered as an alternative security system to traditional authentication and identification methods such as ID card, passwords, code PIN. Biometrics facilitate the process of recognizing a person based on their physiological, behavioral or chemical characteristics

Abdelouahab Attia

attia.abdelouahab@gmail.com

1 LMSE Laboratory, Mohamed El Bachir El Ibrahimi University of Bordj Bou Arreridj, 34000 Bordj Bou Arreridj, Algeria

2 State University of New York Polytechnic Institute, Albany, NY, USA

3 Computer Science Department, Mohamed El Bachir El Ibrahimi University of Bordj Bou Arreridj, 34000 Bordj Bou Arreridj, Algeria

4 Image Team GREYC-CNRS UMR, University of Caen, Caen, France
(Adeoye 2010). Numerous biometric traits have been used in diverse applications ranging from border crossing to mobile authentication (Zhang et al. 2018; Akhtar et al. 2011a, b). In fact, many different biometric traits have been investigated widely such as fingerprint, iris, ear, finger knuckle print, palm print, face etc. (Chaa et al. 2017; Jaswal et al. 2017a). Recently, finger knuckle print (FKP) (Cappelli et al. 2010), which is included in the hand based biometric traits, have been studied in order to improve the consistent authentication system with higher accuracy (Jaswal et al. 2016). FKP has distinctive anatomical structures that can be recorded with low cost and small size imaging devices without using an extra hardware (Cappelli et al. 2010).

Generally, FKP biometrics system can be operated either in identification or verification mode. In identification mode, the given FKP sample is compared with all the available samples to determine the true identity of the subject. In verification mode, the given FKP sample is compared with samples of the claimed identity to determine whether it belongs to the same person. In addition, the FKP verification task is more difficult than the FKP identification because in matching stage it is required to give a global threshold in order to make a decision (Zhang et al. 2011; Aoyama et al. 2014). 
The FKP recognition system over recent years has been attracting considerable attention of researchers. The first team of researchers who introduced the use of finger knuckle surface in biometric systems are Woodard and Flynn (2005). Ferrer et al. (2005) have proposed a ridge features-based algorithm that extracted ridge features from FKP images and evaluated their similarity by applying Hidden Markov Model (HMM) or Support Vector Machine (SVM). Zeinali et al. (2014) have proposed a FKP recognition system, where directional filter bank (DFB) was used to extract feature followed by linear discriminate analysis (LDA) to reduce the dimensionality of the large feature vector. Attia et al. (2018) studied bank of binarized statistical image features (B-BSIF) on FKP trait. Zhang et al. (2012) developed a new computation framework focused on mounting new efficient feature extraction method for FKP recognition. The authors analyzed three commonly used local features, the local orientation, the local phase, and the phase congruency systematically. Also, they presented a method for computing all features efficiently using the phase congruency. Moreover, Hammouche et al. (2020) have proposed a new system for FKP authenticate based on phase congruency with Gabor Filter bank. Heidari and Chalechale (2020) introduced a novel FKP biometric system, where the feature extraction is combination of the entropy-based pattern histogram (EPH) and a set of statistical texture features (SSTF). The genetic algorithm (GA) was employed to locate the superior features among the extracted features. This has been tested on PolyU FKP dataset. While, Muthukumar and Kavipriya (2019) investigated Gabor feature with SVM classifier for FKP identification system. Singh and Kant (2019) have designed a multimodal biometric system based on FKP and iris traits for person authentication, where the PCA method has been used for feature extraction with the Neuro fuzzy neural network (NFNN) classifier in identification step. Malarvizhi et al. (2019) proposed a system named adaptive fuzzy genetic algorithm (AFGA) for Biometric authentication. Wang et al. (2014) investigated depth neural network for finger print classification. Lately, deep learning (i.e., deep neural networks) (Angelov and Soares 2020) have been explored for classification (Gu and Angelov 2018; Sargano et al. 2017) and FKP based biometrics (Chalabi et al. 2020). For instance, Qian et al. (2016) have proposed novel biometric image feature representation technique for FKP, known as deep gradient information (DGI). Jaswal et al. (2017b) considered a new FKP based biometric system that extracted the Region Of Interest (ROI) of FKP trait. Then, the ROI image has been improved and transformed by the invented several methods including, Bubble Ordinal Pattern (BOP), Star Ordinal Pattern (SOP), and Image Ray Transform (IRT). Furthermore, a new Deep Matching method has been used in the matching stage. Zhai et al. (2018) have been planned a new batch-normalized Convolutional Neural
Network (CNN) for FKP authentication system. The data augmentation techniques of random histogram equalization and dropout layer have been implemented to prevent over fitting during training of the proposed scheme.

Thapar et al. (2019) designed a scheme based on deep learning named FKIMNet for Finger Dorsal Image Matching Network. Chlaoua et al. (2019) have investigated a simple deep learning method named principal component analysis Network (PCANet) with SVM classifier. In the proposed scheme, PCA has been used to learn two-stages of filter banks then a simple binary hashing and block histograms for clustering of feature vectors. The output features are finally fed to classification step.

This paper introduces a new FKP system for person authentication based on multilayer deep rule-based classifier. The DRB Classifier is a novel approach that has not been applied before on a biometric system. Further, DRB generates a set of self-organized, transparent IF...THEN FRB (Fuzzy Rule Based) system structure. Each IF...THEN... fuzzy rule of the DRB system contains a various number of prototypes (templates stored in database) that are not pre-determined, recognized via a fully independent, online, non-iterative and non-parametric training process. Also, to their simplicity, the used technique provides a reasonable prototype (templates stored in database), which are then fed to fuzzy rules based of DRB classifier to determine whether the user is genuine or impostor.

The rest of this paper is organized as follows. Section 2 describes the architecture of the DRB classifier. The proposed FKP recognition methodology is outlined in Sect. 3. Section 4 presents the experimental results. The conclusions and future work are given in Sect. 5 .

\section{The DRB classifier architecture and algorithm}

The presented system consists of four principle stages: preprocessing of FKP trait, feature extraction, (training) the DRB classification scheme and decision making. These stages are explained in detail in the following section.

\subsection{The architecture of DRB classifier}

The architecture of DRB classifier was first proposed by Angelov and $\mathrm{Gu}$ (2018). Figure 1 depicts the architecture of DRB classifier. In Fig. 1, it can be seen that the DRB classifier consists of four layers: (i) pre-processing block-the pre-processing block of the DRB classifier generally requires just the basic and commonly used pre-processing methods such as normalization, scaling, rotation and image segmentation; (ii) feature extraction layer - the feature extraction layer of the DRB classifier can use (any) diverse type of feature 


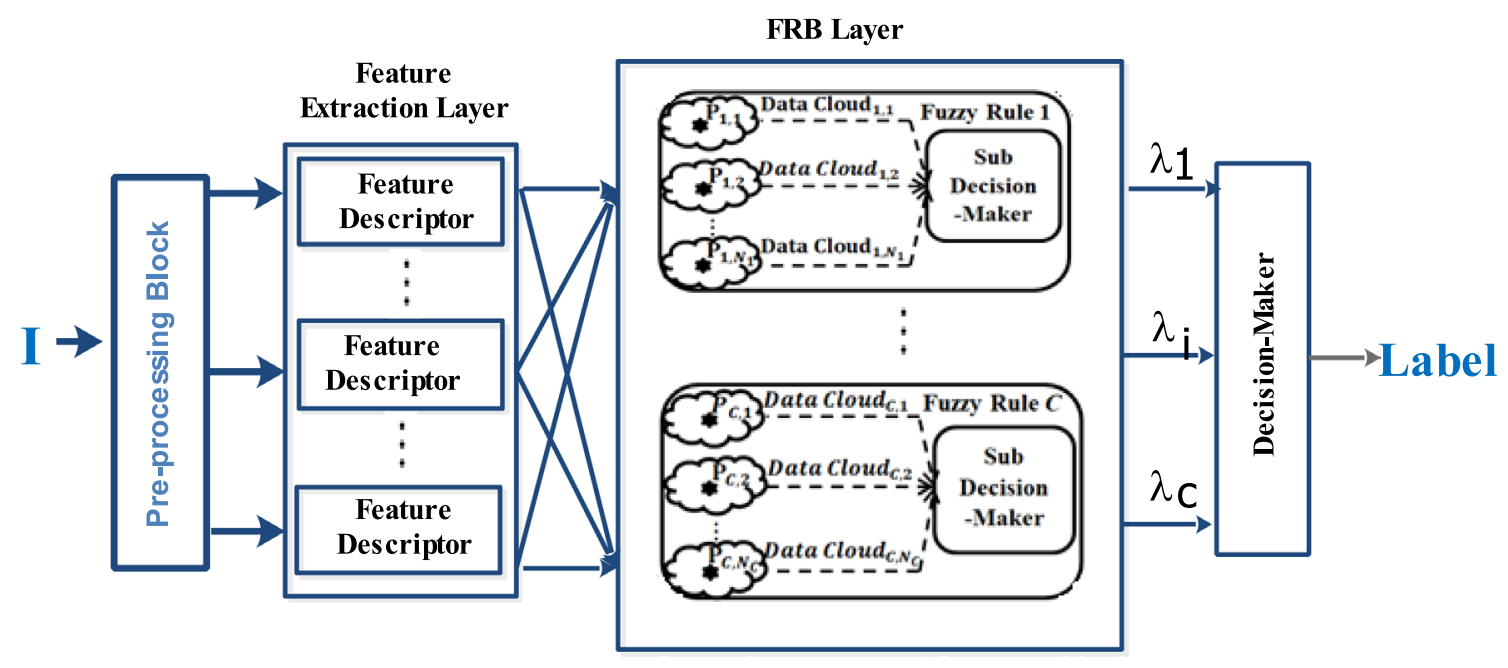

Fig. 1 General architecture of the DRB classifier (Angelov and Gu 2018)

descriptors, which are used in the field of biometric systems. The feature descriptors have some advantages and some limitations (Soltanpour et al. 2017); (iii) fuzzy rule based layer-massively parallel ensemble of highly interpretable IF...THEN... rules. These rules are based on the in parallel self-developing fuzzy rule-based models (Angelov and Yager 2012; Gu et al. 2018) that has been also expressed in Angelov and $\mathrm{Gu}$ (2017) by singletons in the consequent part (0-order models); and (iv) the decision-maker layer-its role is to decide the class label that won based on the partial suggestions of the massively parallel local/sub-decision makers as per IF...THEN... rule/per class. This layer is only used during the validation stage and it applies the "winner-takesall" principle. As a result, one can see that the proposed
DRB classifier actually utilizes a two-stage decision-making structure. The validation process is described in Sect. 3.

\subsection{The DRB classifier algorithm}

The general architecture of the DRB classifier is illustrated in Fig. 1. The DRB classifier algorithm consists of four steps, which are detailed below. Before that, for simplicity, we describe the key notations used in DRB classifier algorithm in Table 1 (Angelov and Gu 2018). For the interested reader, the source code for DRB classifier algorithm can find at (https://www.mathworks.com/matlabcentral/fileexchan ge/67772-deep-rule-based-classifier).

Main steps of DRB classifier algorithm:
Table 1 Description of the key notations used in the algorithm

\begin{tabular}{ll}
\hline Notations & Description \\
\hline $\mathrm{C}$ & The number of classes in dataset \\
$\mathrm{d}$ & The dimensionality of the feature vector \\
$\mathrm{I}$ & The number of the observed training images/current time instance \\
$\mathrm{X}$ & A single image \\
$N_{c}$ & The corresponding feature vector of I \\
$\mu_{c}$ & The number of identified prototypes of the $c$ th class \\
$\mathrm{I}_{c, \mathrm{k}}$ & The global mean of feature vectors of the training images of the $c$ th class \\
$x_{c, k}$ & The $k$ th training image of the $c$ th class \\
$\mathrm{P}_{c, i}$ & The corresponding feature vector of $\mathrm{I}_{c, \mathrm{k}}$ \\
$P_{c, i}$ & The $i$ th prototype of the $c$ th class \\
$S_{c, i}$ & The mean of feature vectors of the training images associated with $\mathbf{P}_{c, i}$ \\
$r_{c, i}$ & The number of training images associated with $\mathbf{P}_{c, i}$ \\
$\chi_{C}$ & The radius of the area of influence of $d a t a c l o u d$ associated with $\mathbf{P}_{c, i}$ \\
\hline
\end{tabular}


Step 1. Pre-processing block: DRB classifier generally requires just the basic and commonly used preprocessing methods, including normalization, scaling, and rotation and image segmentation.

Step 2. Feature extraction layer: DRB classifier can employ diverse type of feature descriptors.

Step 3. Massively Parallel Fuzzy Rule Base (FRB) layer: This step is based on four stages, i.e., initialization, preparation, update of the system, and Fuzzy rules generation.

\section{Stage 0: Initialization}

Initialize all parameters of DRB classifier algorithm such as:

$$
D\left(P_{c, i}\right)=\frac{1}{1+\left\|C-\mu_{c}\right\|^{2} / \sigma_{c}^{2}}
$$

\section{Stage 2: Updating system}

After preparation stage, for each image of stage 1, DRB algorithm checks two conditions to update their parameters. If condition one is verified, add a new data cloud else find the nearest prototype $P_{c, n}$ corresponding to $I_{c, k}$ and go to the condition two. For the condition two, if it is verified Update $P_{c, n}, S_{c, n}$, and $r_{c, n}^{2}$. If condition two is not satisfied add a new data cloud.

\section{Condition 1}

$$
\begin{aligned}
& \text { IF } \\
& \left(D\left(I_{c, k}\right)>_{j=1,2, \ldots \ldots . . N c}^{\max }\left(D\left(P_{c, i}\right)\right)\right) O R\left(D\left(I_{c, k}\right)<_{j=1,2, \ldots \ldots . . N c}^{\min }\left(D\left(P_{c, i}\right)\right)\right) \\
& \text { Then } \\
& I_{c, k} \text { Is new prototype } \\
& \text { Else } \\
& \begin{array}{c}
N_{c} \leftarrow N_{c}+1 ; \\
\mathbf{P}_{c, N c} \leftarrow I_{c, k} ; P_{c, N_{c}} \leftarrow \bar{x}_{c, k} ; S_{c, N c} \leftarrow 1 ; r_{c, N c} \leftarrow r_{0}
\end{array} \\
& 1,2, \ldots, N_{c} \\
& \text { Find } P_{c, n} \text { by } P_{c, n}=\operatorname{argmin}\left(\left\|\bar{X}_{c, k}-P_{c, j}\right\|\right) \text { where }=
\end{aligned}
$$

\section{Condition 2}

$$
\begin{aligned}
& \text { IF } \left.\left(\left\|\bar{x}_{c, k}-P_{c, n}\right\|\right)<r_{c, N c}\right) \text { Then } \\
& \left(I_{c, k} \text { is assigned to } P_{c, n}\right) \\
& \text { Else } \\
& \quad N_{c} \leftarrow N_{c}+1 ; \mathbf{P}_{c, N c} \leftarrow I_{c, k} ; P_{c, N_{c}} \leftarrow \bar{x}_{c, k} ; S_{c, N c} \leftarrow \\
& 1 ; r_{c, N c} \leftarrow r_{0} \underset{\text { Endif }}{\text { Endif }}
\end{aligned}
$$

$k \leftarrow 1 ; \mu c \leftarrow \bar{x}_{c l} ; N_{c} \leftarrow 1 ; P_{c, N c} \leftarrow I_{c, 1} ; P_{c, N c} \leftarrow \bar{x}_{c l} ; S_{c, N c} \leftarrow 1 ; r_{c, N c} \leftarrow r_{0} ;$

\section{Stage 1: Preparation}

For each image $\mathrm{I}_{\mathrm{c}, \mathrm{k}}: \bar{x}_{c, k}, \mu_{c}$, and $D\left(P_{c, i}\right)$ is calculated such as:

$\bar{x}_{c, k}=\frac{x_{c, k}}{\left\|x_{c, k}\right\|} ; \mu_{c}=\frac{k-1}{k} \mu_{c}+\bar{x}_{c, k}$

\section{Stage 3: Fuzzy rules generation}

\section{Generate rule type}

Rule $_{\mathbf{c}}: \operatorname{IF}\left(I \sim P_{c, 1}\right)$ OR ... OR $\left(I \sim P_{c, N c}\right) \operatorname{THEN}($ classe $c)$

Stage 4: Decision maker layer

After the generation of $\mathrm{C}$ fuzzy rules to the $\mathrm{C}$ classes, for each image $\mathrm{I}$ in test data, generate a score of confidence $\chi_{\boldsymbol{c}}(\boldsymbol{I})$ based on the feature vector of image I such as:

$$
-\chi_{c}(I)=\arg \max \left(\exp \left(-\left\|x-P_{c, j}\right\|^{2}\right)\right), j=1,2, \ldots, N_{c} \text {. }
$$

- Identified labelby:Label$(I)=\arg \max \left(\chi_{c}(I)\right), c=1,2 \ldots, c$. 
Fig. 2 The architecture of the proposed recognition system
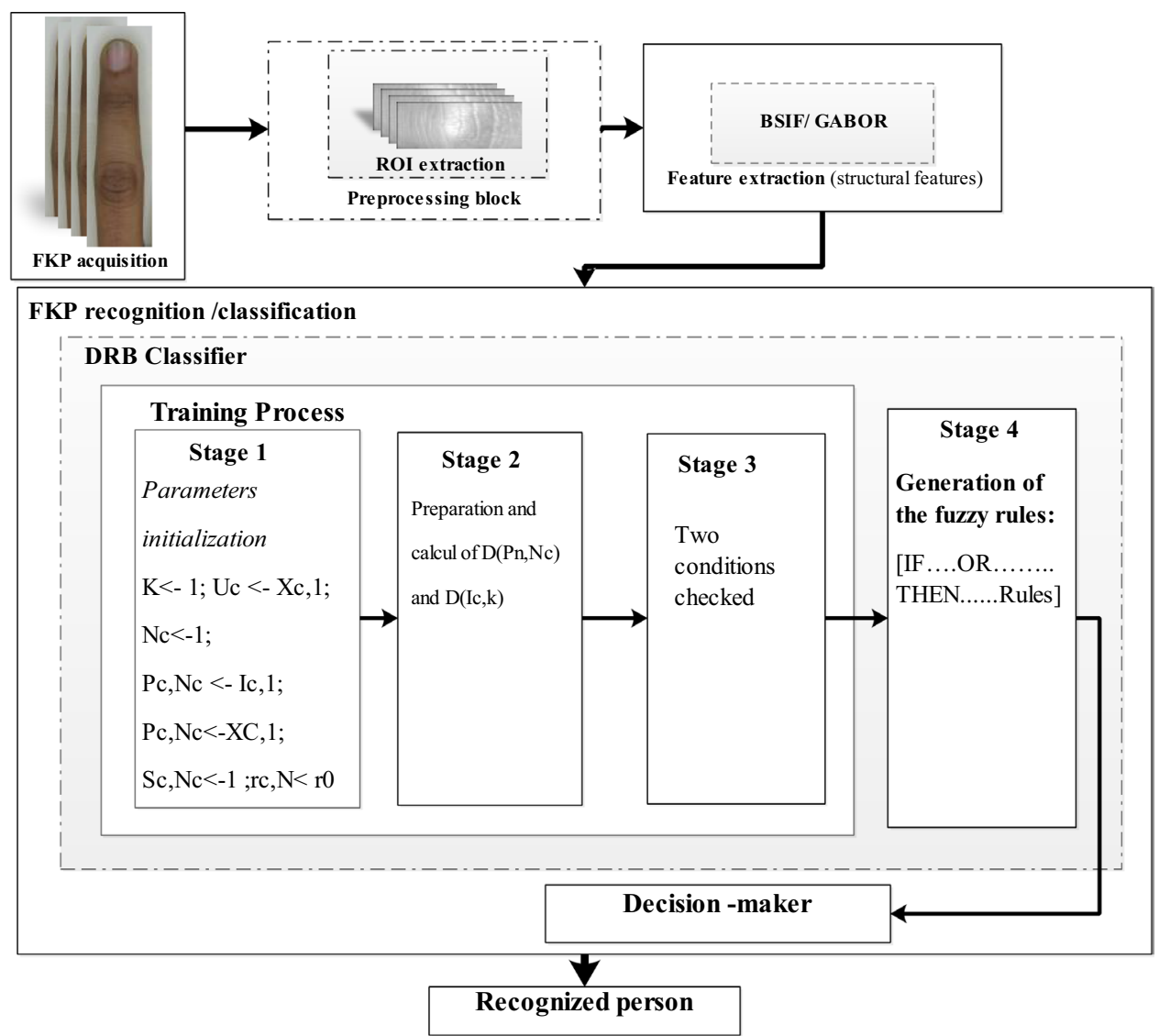

\section{The proposed FKP biometric system based on DRB classifier}

The global architecture of the proposed FKP biometric system, including BSIF and Gabor based methods as main feature extractions and DRB classifier, is illustrated in Fig. 2, the global architecture of the proposed method is composed of a sequence of four main phases starting with the acquisition of FKP images, ROI extraction operations, and then the extraction of descriptive features that are fed to the proposed classifier multilayer deep rule based (DRB) to determine whether the user is genuine or impostor.

\subsection{ROI extraction}

The extraction the region of interest ROI for FKP (Jaswal et al. 2017a) consists of few steps. Firstly, the Gaussian smoothing operation is applied to the original image, and then the smoothed image is down sampled to $150 \mathrm{dpi}$. Secondly, the $\mathrm{x}$-axis of the coordinate system fitted from the bottom boundary of the finger is determined; note that the bottom boundary of the finger can be easily extracted by a canny edge detector. Thirdly, the $y$-axis of the coordinate system is selected by applying a canny edge detector on the cropped sub-image that is previously extracted from the original image base on $\mathrm{x}$-axis, then the convex direction coding scheme is found. Finally, the ROI is extracted,

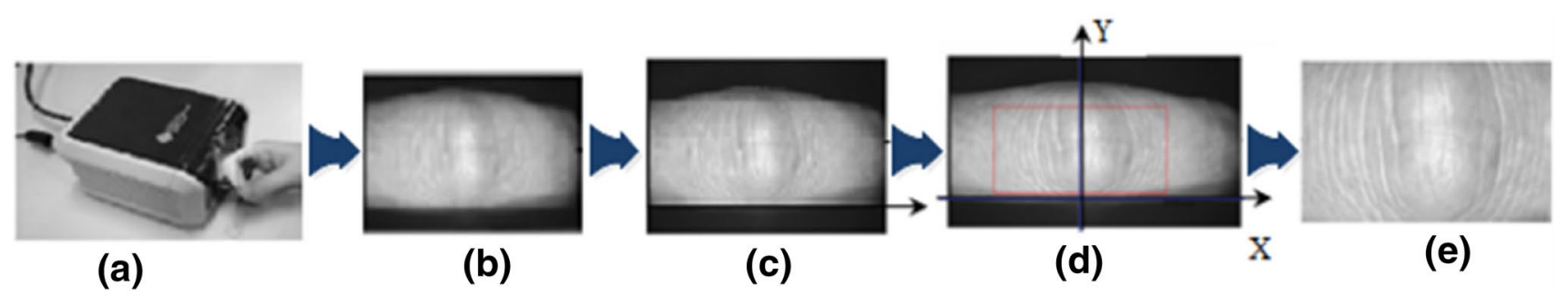

Fig. 3 The steps of extraction of FKP ROI image 
where the rectangle indicates the area of the ROI, which will be extracted and used further user recognition as shown in Fig. 3.

\subsection{Feature extraction}

Feature extraction is another important stage. There are various kinds of feature descriptors presented in the literature. However, in this paper we chose the BSIF descriptor (binarized statistical image features) and Gabor feature extraction because these two have been successfully employed widely for object detection and recognition.

\subsubsection{BSIF descriptor (binarized statistical image features)}

Binarized statistical image feature (BSIF) is a textural local descriptor presented in Kannala and Rahtu (2012a). This feature descriptor basically utilizes a set of filters of fixed size that describes the neighborhood configuration of the central pixel. BSIF filters a given image $J$ of size $m \times n$ with a set of filters $\varphi_{i}^{k \times k}$ then the responses are binarized. The filter response is obtained as follows:

$r_{i}=\sum_{m, n} \varphi_{i}^{k \times k} J(m, n)$

Fig. 4 a Sample of the FKP ROI image, $\mathbf{b}$ BSIF filter with a size $11 \times 11$ and of length 12 , c BSIF features $\mathbf{d}$ final BSIF where $\varphi_{i}^{k \times k}$ is a linear filter of size $k$ and $i=\{1,2, \ldots, n\}$ denotes the number of statistically independent filters whose response can be computed together and binarized to obtain the binary string as follows (Kannala and Rahtu 2012b):

$b_{i}= \begin{cases}1 & \text { if } r_{i}<0 \\ 0 & \text { otherwise }\end{cases}$

Lastly, the BSIF features are obtained as the histogram of pixel's binary codes that can effectively characterizes the texture components in the FKP image. There are two important factors in BSIF descriptor namely: the filter size $k$ and the filter length $(n)$. The corresponding BSIF code (https://www.ee.oulu.fi/ jkannala/bsif/bsif.html) depth and intensity images are shown in Fig. 4. Thus, Fig. 4a indicates the input ROI FKP image. Figure $4 b$ shows the learned BSIF filter with a size $11 \times 11$ and of length 12 . Figure $4 \mathrm{c}$ shows the results of the individual convolution of the ROI FKP image with BSIF filter. Figure 4d shows the final BSIF feature encoded.

\subsubsection{Gabor feature extraction}

Gabor wavelet is another useful tool in image analysis and computer vision thanks to its optimal localization properties in spatial analysis as well as frequency domain. Broadly speaking, the family of 2D Gabor wavelets (https://es.mathw

(a)

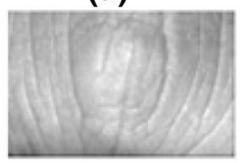

(b)
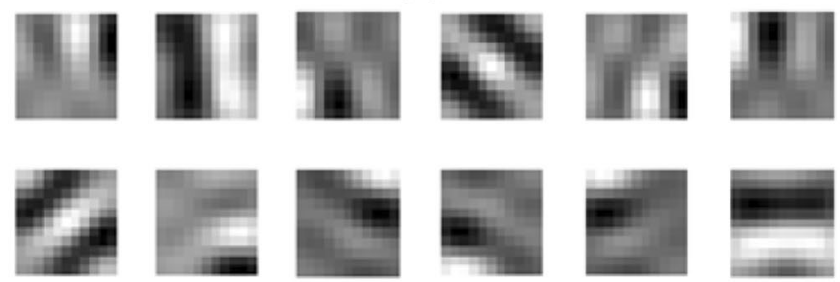

(c)
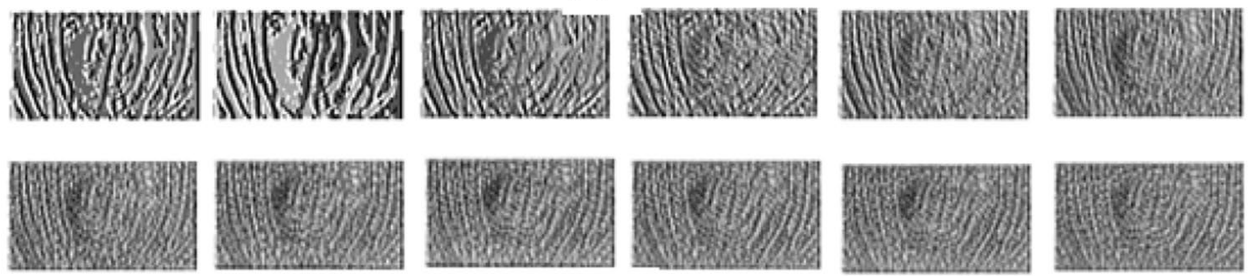

(d)
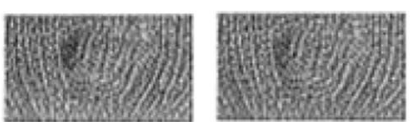
orks.com/matlabcentral/fileexchange/44630-gabor-featureextraction) can be described in the spatial domain (Liu and Wechsler 2002; Shen and Bai 2006) as follows:

$H_{\mu, \vartheta}=\frac{f_{\mu}^{2}}{\pi n \lambda} \exp \left[-\left(\frac{f_{\mu}^{2}}{n^{2}}\right) x_{p}^{2}-\left(\frac{f_{\mu}^{2}}{\lambda^{2}}\right) y_{p}^{2}\right] \exp \left(j 2 \pi x_{p}\right)$

where $\quad x_{p}=x \cdot \cos \left(\theta_{\vartheta}\right)+y \bullet \sin \left(\theta_{\vartheta}\right), y_{p}=-x \cdot \sin \left(\theta_{\vartheta}\right)$ $+y \bullet \cos \left(\theta_{\vartheta}\right), f_{\mu}=f_{\max } / 2^{(\mu / 2)}$ and $\theta_{\vartheta}=\nu \pi / 8$ with $f_{\mu}$ and $f_{\text {max }}$ are the center and the maximal frequency of Gabor filter, respectively, and $\theta_{\vartheta}$ denotes the orientation. The parameters $\eta$ and $\lambda$ explain the size of the Gaussian envelope along $\mathrm{x}$-axis and $\mathrm{y}$-axis correspondingly. The factors of the Gabor filter bank are experimentally selected as: $f_{\max }=0.25$ and $\lambda=\eta=\sqrt{2}$. The Gabor representation of a J-image can be done by convolving this image with the bank of Gabor filter ( 5 scales, 8 orientations) as defined by:

$Y_{\mu, \vartheta}=J(x, y) * H_{\mu, \vartheta}$

where $J(x, y)$ is considered as complex structure of two images real and imaginary denoted as R-image or I-image and $*$ is the product convolution. The output $Y_{\mu, \vartheta}$ has the complex structure; the augmented magnitude feature vector of the J-image is extracted.

First for each J-image, special Gabor filter bank is applied with 5 scales $(\mu)$ and 8 orientations $(v)$ to obtain 40 filtered images. Then, the calculation of 40 magnitudes responses from 40 filtered image where each magnitude response is then down-sampling by a factor $\rho=64$ and preprocessed by normalization to zero mean with unit variance Fig. 5 shows an example. Lastly, each down-sampled magnitude response $M_{\mu, \vartheta}$ is reorganized into a vector $W_{\mu, \vartheta}$ by scan columns. The 40 vectors are concatenated to construct the feature vector of R-image or I-image as it can be seen in the following equation:
$Y=\left[W_{0.0}, W_{0.1}, \cdots, W_{4.7}\right]^{T}$

\subsection{Classification}

Once the selection of the discriminative and pertinent characteristics of each image is done, the different classes are separated, and the decision is made in order to accept the user as genuine or reject as an impostor.

In order to carry out this stage in our system, we have employed Deep Rule-Based (DRB) based classification technique.

\subsubsection{DRB training architecture}

Deep Rule-Based classifier is a process that works with four main phases representing its global system mechanism (Angelov and $\mathrm{Gu} 2018$ ), which are initialization phase, preparation phase, updating phase, fuzzy rules generation phase.

Table 2 Parameters initialization

\begin{tabular}{ll}
\hline Parameter & Description \\
\hline $\mathbf{K} \leftarrow 1 ;$ & $\mathrm{k}$ is the current time instance \\
$\boldsymbol{\mu}_{\mathbf{c}} \leftarrow \mathbf{X}_{\mathbf{c}, 1} ;$ & $\mu_{\mathrm{c}}$ is the global mean of all the observed data \\
of the class c \\
$\mathbf{N}_{\mathbf{c}} \leftarrow 1 ;$ & $\mathrm{N}_{\mathrm{c}}$ is the number of prototypes \\
$\boldsymbol{P}_{c, N_{c}} \leftarrow \mathrm{Ic}, 1 ; \boldsymbol{P}_{\boldsymbol{c}, N_{c}} \leftarrow$ & $P_{c, N_{c}}$ prototypes of the class c \\
$\mathrm{X}_{\mathrm{C}, 1} ;$ & $S_{c, N c}$ number of images of the data cloud \\
$\boldsymbol{S}_{\boldsymbol{c}, N c} \leftarrow 1 ;$ & $r_{c, N c}$ is the radius of the area of the data cloud \\
$\boldsymbol{r}_{\boldsymbol{c}, N c} \leftarrow \boldsymbol{r}_{0}$ & \\
\hline
\end{tabular}

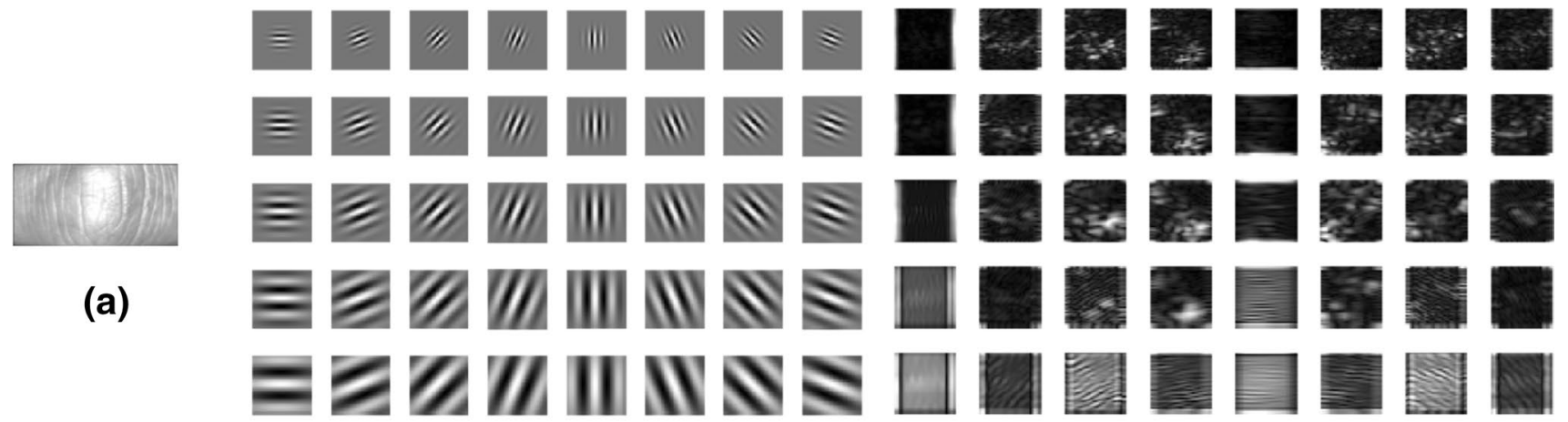

(b) (c)

Fig. 5 a original FKP image, b 40 Gabor filter bank with different scales and orientations, c magnitude of the image filtered by every Gabor filter 


\subsubsection{Initialization phase}

In this phase, the classifier begins with a vector normalization operation $X_{c, 1}$ applied to the global feature vector of the first image of the class " $c$ " $I_{c, 1}$ with an initialization of the different meta-parameters of the system as shown in Table 2:

\subsubsection{Preparation phase}

For all the images of all the classes $I_{c, k}, X_{c, k}$ is the normalization vector applied to its corresponding feature vectors, the global mean $\mu_{\mathrm{c}}$ is updated as shown in the following instruction:

$\mu_{c}=\frac{K-1}{K} \mu_{c}+\frac{1}{K} \bar{X}_{c, k}$

Then, the density of all the existing prototypes $P_{c}, N_{c}$ of the new image $I_{c, k}$ is calculated as denoted by: $\mathrm{D}\left(P_{c, N_{c}}\right)$ and $\mathrm{D}\left(I_{c, k}\right)$.

\subsubsection{Updating stage}

From the previously calculated $\mu_{\mathrm{c}}, \mathrm{D}\left(P_{c, N_{c}}\right)$ and $\mathrm{D}\left(I_{c, k}\right)$, two conditions are identified:

Condition 1: IF $\left[\mathrm{D}\left(I_{c, k}\right)>\max \mathrm{D}\left(P_{c, N_{c}}\right)\right]$ OR $\left[\mathrm{D}\left(I_{c, k}\right.\right.$ )$\left.<\min \mathrm{D}\left(P_{c, N_{c}}\right)\right]$ THEN $\left(I_{c, k}\right)$ is set to a new prototype and initializes a new data cloud.however if this condition is not verified, $\mathrm{P}_{\mathrm{c}, \mathrm{n}}$ look for the nearest prototype to $I_{c, k}$ as shown in Eq. 7:

$P_{c, n}=\arg \min \left(\left\|\bar{X}_{c, k}-P_{c, j}\right\|\right) \quad$ where $j=1,2, \ldots, N_{c}$

where argmin is function that gives the nearest prototype by minimizing the distance between the normalized feature vector denoted by $\bar{X}_{c, k}$ and the mean of feature vectors of the training images $P_{c, j}$.

Condition 2: IF $\left[\left\|X_{c, k}-P_{c, n}\right\|<r_{c, N_{c}}\right]$ THEN $\left[\mathrm{I}_{\mathrm{c}, \mathrm{k}}\right.$ is assigned to $\mathrm{P}_{\mathrm{c}, \mathrm{n}}$ ] ELSE $\left[I_{c, k}\right.$ is out of the area of $\left.P_{c, n}\right]$.

A new data cloud is added, the next image is grabbed at phase 2 (preparation phase) with the processing of all the training samples.

\subsubsection{Fuzzy rules generation stage}

In this last phase, the system generate one Anaya rule based on the identified prototypes

$$
R: I F\left(I \sim P_{c, 1}\right) \text { OR } \ldots \text { OR }\left(I \sim P_{c, N_{c}}\right) \operatorname{THEN}(\text { classc })
$$

where ' 'denotes similarity; I is a particular image with its corresponding vector feature vector $\mathrm{x} ; \mathrm{P}$ is the prototype; $N_{c}$ is the number of prototypes of the $c$ th class;

\section{Experiments}

In this section, we provide an experimental evaluation of the proposed FKP based person recognition system.

\subsection{FKP database}

The proposed method has been tested on the publicly available FKP dataset that provided by Hong Kong Polytechnic University (PolyU) (2010). This database has 7920 images collected from 165 persons with 125 males and 40 females, their age is in the range of 20-50 years old. The images were captured in two sessions, 48 different FKP images of each individual. Four finger types of every person have been collected that are: Left Index Finger (LIF), Left Middle Finger (LMF), Right Index Finger (RIF) and Right Middle Finger (RMF). Every finger type has 6 images in each session. There are total 1980 number of images for each finger type.

\subsection{Experiment results}

In this section, we report two different experiments: Experiment I-where we tested the approach on single modality, and Experiment II-where we tested the approach on Multitrait (Multi modalities) FKP system.

\subsection{Experiment I: single modality}

The main goal of this experiment is to test and evaluate the introduced scheme on FKP single modality (LIF, RIF, RMF, LMF) using DRB classifier with different distances such as Euclidean, Cos, Correlation as well as Gabor filter bank and BSIF descriptor. In this sets of experiments, we highlight the

Table 3 Single modalities results

\begin{tabular}{lllllll}
\hline \multirow{2}{*}{ Modality } & Distance & \multicolumn{2}{l}{ Gabor-DRB } & & \multicolumn{2}{l}{ BSIF-DRB } \\
\cline { 3 - 4 } \cline { 6 - 7 } & & Acc $(\%)$ & EER $(\%)$ & & Acc $(\%)$ & EER $(\%)$ \\
\hline RIF & Euclidean & 93.43 & 5.55 & & 93.94 & 4.20 \\
& Cosine & 93.08 & 5.61 & & 93.43 & 4.10 \\
& Correlation & 93.08 & 5.61 & & 93.08 & 6.63 \\
RMF & Euclidean & 94.09 & 5.21 & & 94.14 & 4.13 \\
& Cosine & 94.19 & 5.10 & & 92.98 & 3.99 \\
& Correlation & 94.19 & 5.10 & & 93.28 & 6.98 \\
LIF & Euclidean & 94.34 & 4.95 & & 92.73 & 4.72 \\
& Cosine & 93.94 & 4.99 & & 91.46 & 5.23 \\
& Correlation & 93.94 & 4.99 & & 91.77 & 8.34 \\
LMF & Euclidean & 93.43 & 5.96 & & 93.54 & 4.19 \\
& Cosine & 93.08 & 5.91 & 92.47 & 4.40 \\
& Correlation & 93.08 & 5.91 & 92.78 & 6.77 \\
\hline
\end{tabular}




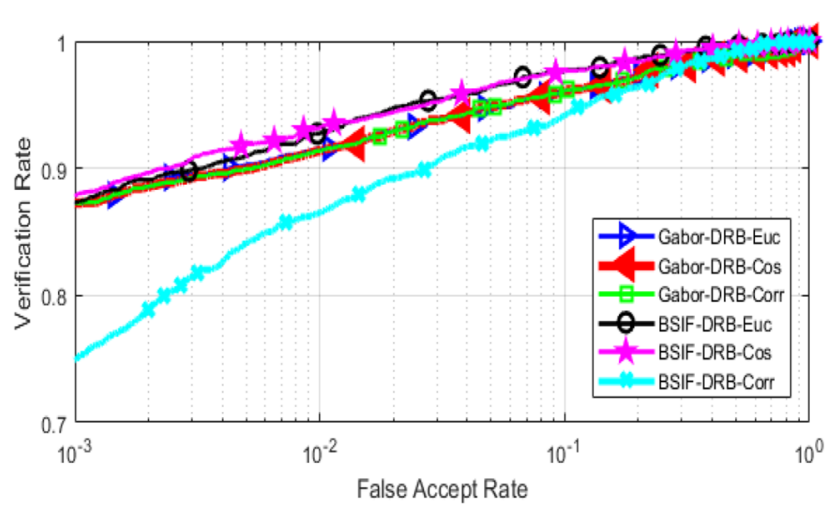

(a)

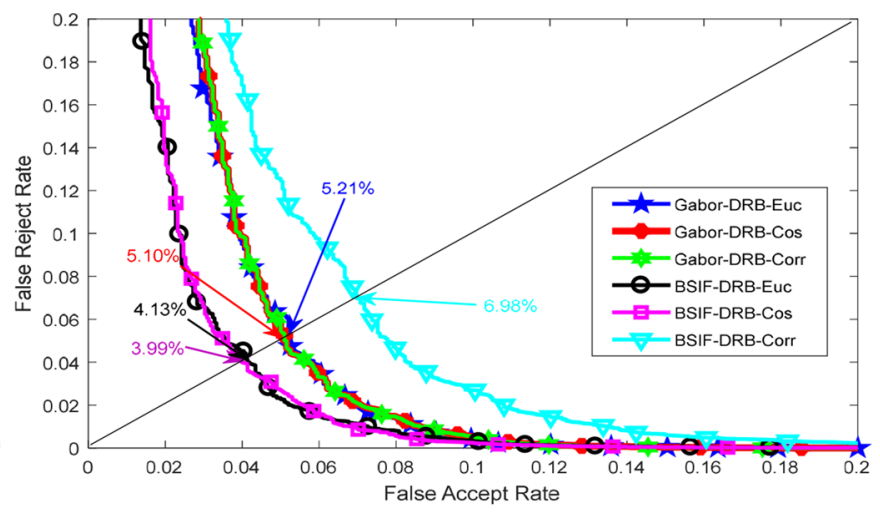

(b)

Fig. 6 RMF modality results: a ROC, b EER curves

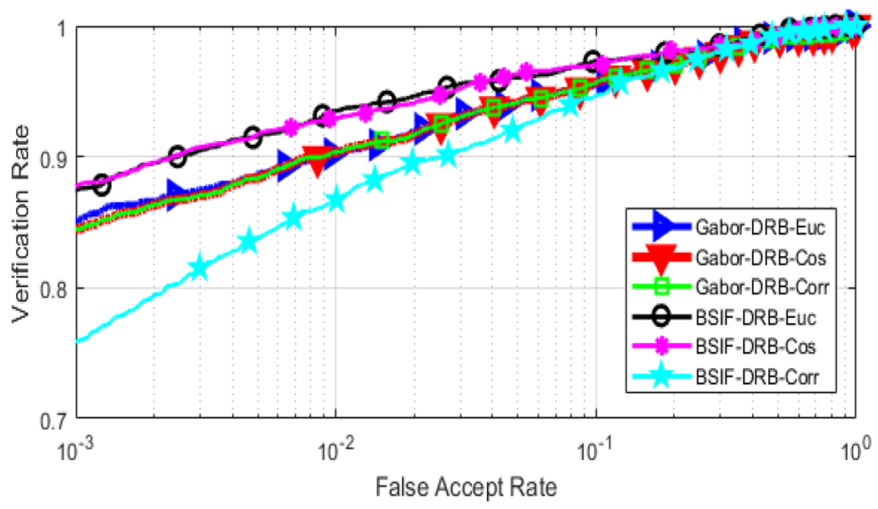

(a)

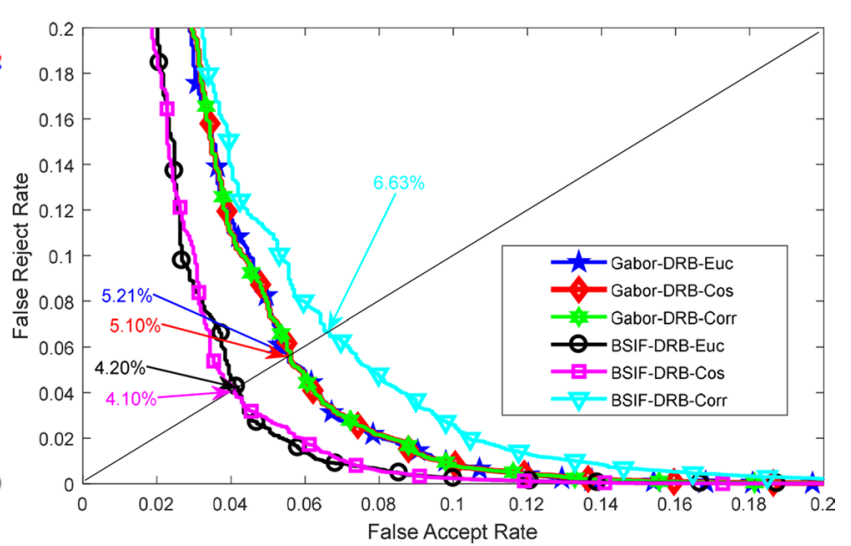

(b)

Fig. 7 RIF modality results: a ROC, b EER curves

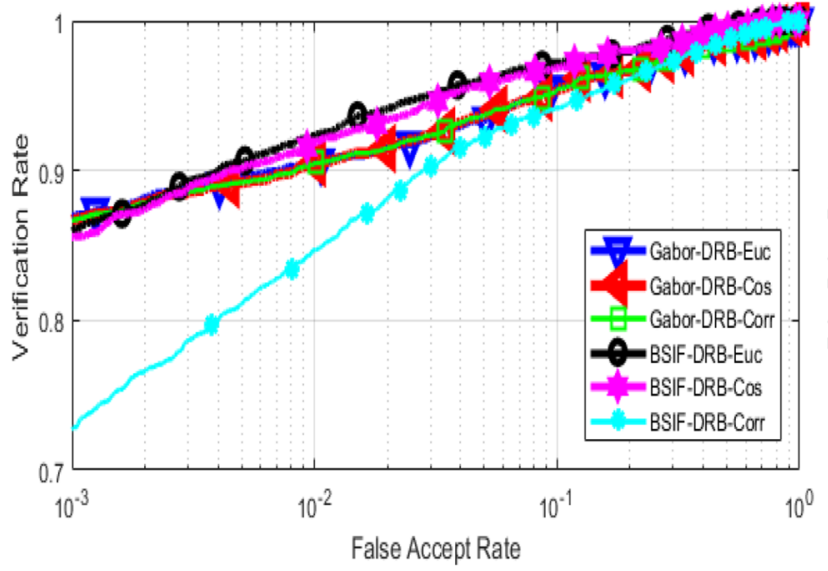

(a)

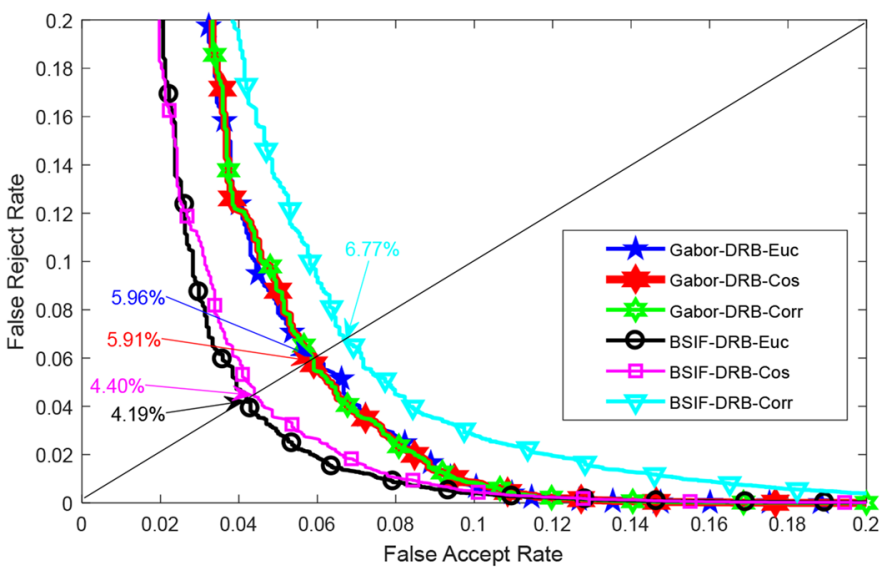

(b)

Fig. 8 LMF modality results: a ROC, b EER curves 


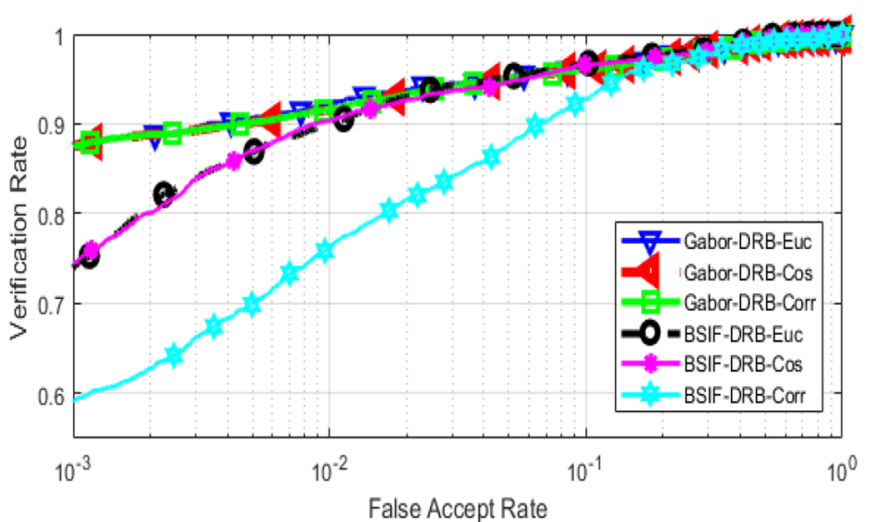

(a)

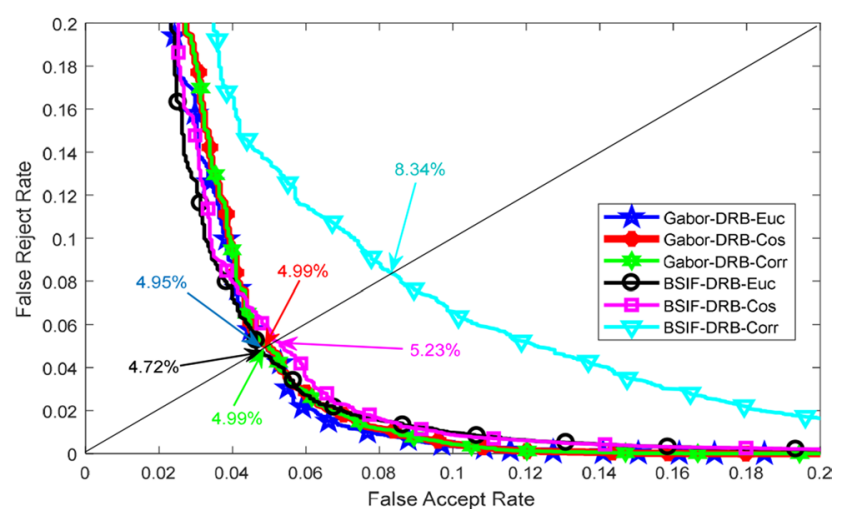

(b)

Fig. 9 LIF modality results: a ROC, b EER curves

Table 4 Comparative of proposed personal recognition method with the existing approaches for FKP using PolyU dataset

\begin{tabular}{llllll}
\hline Identification (Rank-1) & \multicolumn{1}{l}{} \\
\hline References & Year & LMF (\%) & LIF (\%) & RIF (\%) & RMF (\%) \\
\hline El-Tarhouni et al. (2014) & 2014 & 94.70 & 93.80 & 92.20 & 94.80 \\
Shariatmadar and Faez (2013) & 2013 & 95.54 & 94.33 & 95.93 & 96.72 \\
Zeinali et al. (2014) & 2014 & 90.30 & 88.68 & 89.79 & 89.79 \\
Proposed method & 2020 & $\mathbf{9 3 . 5 4}$ & $\mathbf{9 4 . 3 4}$ & $\mathbf{9 3 . 9 4}$ & $\mathbf{9 4 . 1 9}$ \\
\hline
\end{tabular}

Our achieved results are indicated in bold comparison between distances and descriptors that achieve good accuracy.

The results of BSIF-DRB and Gabor-DRB on individual FKP traits are presented in Table 3. We can observe in Table 3 that BSIF-DRB performs better than Gabor-DRB. The proposed method using BSIF-DRB achieves higher accuracy on the RIF, LMF, and RMF, modalities, e.g., on RMF modality the system achieved EER $=4.13 \%$ and accuracy $=94.14 \%$ using Euclidean distance in verification and identification modes, respectively. Gabor-DRB performs better than BISF-DRB on the LIF modality using the three distances.

To further explore our findings, the BSIF-DRB and Gabor-DRB comparison results are also presented in term of EER and ROC curves, which can be seen in Figs. 6, 7, 8, 9. These graphs also report comparison study between the proposed method BSIF-DRB and Gabor-DRB with different distances using FKP modality. The results clearly show that the performance is better when the system uses the features of the BSIF descriptor with Euclidean distance.

To further demonstrate the efficacy of the proposed system, the comparison of the proposed framework has been done with prior works in the literature on individual FKP modalities, as shown in Table 4. The proposed scheme achieves accuracy in range of $91.46 \%$ to $94.34 \%$, which is a good result compared to the existing FKP person recognition
Table 5 Two fingers fusion results using min rule score level fusion

\begin{tabular}{|c|c|c|c|c|c|}
\hline \multirow[t]{2}{*}{ Modality } & \multirow[t]{2}{*}{ Distance } & \multicolumn{2}{|c|}{ Gabor-DRB } & \multicolumn{2}{|c|}{ BSIF-DRB } \\
\hline & & $\operatorname{Acc}(\%)$ & $\operatorname{EER}(\%)$ & $\operatorname{Acc}(\%)$ & $\operatorname{EER}(\%)$ \\
\hline \multirow[t]{3}{*}{ RIF-RMF } & Euclidean & 98.43 & 2.17 & 97.27 & 2.21 \\
\hline & Cosine & 98.48 & 2.21 & 96.52 & 2.67 \\
\hline & Correlation & 98.48 & 2.21 & 95.91 & 3.69 \\
\hline \multirow[t]{3}{*}{ RIF-LMF } & Euclidean & 97.63 & 2.93 & 96.82 & 1.97 \\
\hline & Cosine & 97.42 & 2.83 & 96.52 & 2.27 \\
\hline & Correlation & 97.42 & 2.83 & 96.67 & 2.70 \\
\hline \multirow[t]{3}{*}{ RIF-LIF } & Euclidean & 98.28 & 2.28 & 96.67 & 2.67 \\
\hline & Cosine & 98.08 & 2.51 & 95.76 & 2.84 \\
\hline & Correlation & 98.08 & 2.51 & 94.95 & 2.51 \\
\hline \multirow[t]{3}{*}{ RMF-LMF } & Euclidean & 98.08 & 2.27 & 97.37 & 1.99 \\
\hline & Cosine & 97.63 & 2.37 & 96.82 & 2.16 \\
\hline & Correlation & 97.63 & 2.37 & 96.36 & 2.43 \\
\hline \multirow[t]{3}{*}{ RMF-LIF } & Euclidean & 98.08 & 2.24 & 97.32 & 2.68 \\
\hline & Cosine & 97.73 & 2.42 & 96.82 & 2.42 \\
\hline & Correlation & 97.73 & 2.42 & 95.61 & 3.54 \\
\hline \multirow[t]{3}{*}{ LMF-LIF } & Euclidean & 96.87 & 2.13 & 96.62 & 2.84 \\
\hline & Cosine & 96.72 & 2.29 & 95.86 & 2.86 \\
\hline & Correlation & 96.72 & 2.29 & 95.05 & 3.02 \\
\hline
\end{tabular}

systems. For instance, the proposed method on LIF modality is able to attain $94.34 \%$ in identification mode. While, FKP 
Table 6 Two finger fusion results using sum rule score level fusion

\begin{tabular}{lllllll}
\hline Modality & Distance & \multicolumn{2}{l}{ Gabor-DRB } & & \multicolumn{2}{l}{ BSIF-DRB } \\
\cline { 3 - 4 } \cline { 6 - 7 } \cline { 6 - 7 } RIF-RMF & Euclidean & 98.03 & 1.84 & & 97.63 & 2.37 \\
& Cosine & 97.32 & 2.13 & & 97.17 & 2.63 \\
& Correlation & 97.32 & 2.13 & & 97.17 & 2.93 \\
RIF-LMF & Euclidean & 97.53 & 2.34 & & 97.58 & 2.11 \\
& Cosine & 96.52 & 2.63 & & 96.62 & 2.74 \\
& Correlation & 96.52 & 2.63 & & 96.52 & 2.24 \\
RIF-LIF & Euclidean & 98.13 & 1.44 & & 97.93 & 2.43 \\
& Cosine & 97.78 & 1.98 & & 96.82 & 2.82 \\
& Correlation & 97.78 & 1.98 & & 97.37 & 1.99 \\
RMF-LMF & Euclidean & 97.78 & 1.92 & & 98.32 & 1.82 \\
& Cosine & 97.42 & 2.24 & & 97.42 & 2.07 \\
& Correlation & 97.42 & 2.24 & & 96.72 & 2.69 \\
RMF-LIF & Euclidean & 97.73 & 1.79 & & 98.13 & 1.47 \\
& Cosine & 97.22 & 2.44 & & 97.07 & 2.83 \\
& Correlation & 97.22 & 2.44 & & 97.12 & 2.69 \\
LMF-LIF & Euclidean & 97.32 & 2.08 & & 97.12 & 2.01 \\
& Cosine & 96.92 & 2.34 & 96.92 & 2.13 \\
& Correlation & 96.92 & 2.34 & 96.21 & 2.57 \\
\hline
\end{tabular}

Table 7 Three finger and all fingers fusion results using min rule score level fusion

\begin{tabular}{|c|c|c|c|c|c|}
\hline \multirow[t]{2}{*}{ Modality } & \multirow[t]{2}{*}{ Distance } & \multicolumn{2}{|c|}{ Gabor-DRB } & \multicolumn{2}{|c|}{ BSIF-DRB } \\
\hline & & $\operatorname{Acc}(\%)$ & EER (\%) & Acc (\%) & EER (\%) \\
\hline \multirow{3}{*}{$\begin{array}{l}\text { RIF-RMF- } \\
\text { LMF }\end{array}$} & Euclidean & 99.34 & 1.17 & 98.48 & 1.51 \\
\hline & Cosine & 98.99 & 1.22 & 97.68 & 1.58 \\
\hline & Correlation & 98.99 & 1.22 & 97.53 & 2.22 \\
\hline \multirow[t]{3}{*}{ RIF-RMF-LIF } & Euclidean & 99.29 & 1.47 & 98.13 & 1.17 \\
\hline & Cosine & 99.29 & 1.41 & 97.53 & 1.97 \\
\hline & Correlation & 99.29 & 1.41 & 96.31 & 2.38 \\
\hline \multirow[t]{3}{*}{ RIF-LMF-LIF } & Euclidean & 98.48 & 1.92 & 98.48 & 1.82 \\
\hline & Cosine & 98.33 & 1.87 & 97.53 & 1.67 \\
\hline & Correlation & 98.33 & 1.87 & 96.57 & 2.44 \\
\hline \multirow{3}{*}{$\begin{array}{l}\text { RMF-LMF- } \\
\text { LIF }\end{array}$} & Euclidean & 99.14 & 1.51 & 98.64 & 1.92 \\
\hline & Cosine & 98.64 & 1.56 & 98.03 & 1.58 \\
\hline & Correlation & 98.64 & 1.56 & 97.02 & 2.61 \\
\hline \multirow[t]{3}{*}{ All finger } & Euclidean & 99.65 & 0.91 & 99.09 & 1.51 \\
\hline & Cosine & 99.39 & 0.91 & 98.28 & 1.42 \\
\hline & Correlation & 99.39 & 0.91 & 97.42 & 1.69 \\
\hline
\end{tabular}

based person recognition systems proposed in El-Tarhouni et al. (2014) achieved $93.80 \%$.
Table 8 Three finger and all fingers fusion results using sum rule score level fusion

\begin{tabular}{|c|c|c|c|c|c|}
\hline \multirow[t]{2}{*}{ Modality } & \multirow[t]{2}{*}{ Distance } & \multicolumn{2}{|c|}{ Gabor-DRB } & \multicolumn{2}{|c|}{ BSIF-DRB } \\
\hline & & $\operatorname{Acc}(\%)$ & $\operatorname{EER}(\%)$ & $\operatorname{Acc}(\%)$ & $\operatorname{EER}(\%)$ \\
\hline \multirow{3}{*}{$\begin{array}{l}\text { RIF-RMF- } \\
\text { LMF }\end{array}$} & Euclidean & 98.89 & 1.07 & 98.94 & 1.47 \\
\hline & Cosine & 98.33 & 1.43 & 98.69 & 1.47 \\
\hline & Correlation & 98.33 & 1.43 & 98.59 & 1.69 \\
\hline \multirow[t]{3}{*}{ RIF-RMF-LIF } & Euclidean & 98.84 & 1.12 & 98.99 & 1.52 \\
\hline & Cosine & 98.28 & 1.57 & 98.84 & 1.92 \\
\hline & Correlation & 98.28 & 1.57 & 98.79 & 1.68 \\
\hline \multirow[t]{3}{*}{ RIF-LMF-LIF } & Euclidean & 98.69 & 1.19 & 99.19 & 1.81 \\
\hline & Cosine & 98.13 & 1.82 & 98.28 & 1.12 \\
\hline & Correlation & 98.13 & 1.82 & 98.43 & 1.67 \\
\hline \multirow{3}{*}{$\begin{array}{l}\text { RMF-LMF- } \\
\text { LIF }\end{array}$} & Euclidean & 98.38 & 1.27 & 99.24 & 1.02 \\
\hline & Cosine & 97.98 & 1.93 & 98.84 & 1.87 \\
\hline & Correlation & 97.98 & 1.93 & 98.38 & 1.14 \\
\hline \multirow[t]{3}{*}{ All finger } & Euclidean & 99.24 & 0.82 & 99.65 & 0.19 \\
\hline & Cosine & 98.64 & 1.58 & 99.44 & 0.40 \\
\hline & Correlation & 98.64 & 1.58 & 98.89 & 1.48 \\
\hline
\end{tabular}

\subsection{Experiment II: multi-traits (multimodal) biometrics system}

Our main aim for this experiment is to investigate the performance of proposed system in the case of information fusion, given that multimodal systems that combine information from different sources are able to over some limitations (e.g., accuracy, noise, etc.) of the unimodal biometric systems. Thus, further tests were carried out, where we studied different scenarios when the information presented by different finger types (LIF, LMF, RIF, and RMF) modality are integrated.

The experiments were performed using level score fusion, where the information were fused by applying two score rules min rule and sum rule. We distinguish three experimental investigation were conducted by (i) fusing only two types of fingers as shown in Tables 5, 6, (ii) three types fingers as revealed in Tables 7, 8, (iii) all types of fingers as it can be seen in Tables 7, 8. Finally, a comparison with existing FKP multimodal methods in reported in Table 9.

\subsubsection{Experiment 1: two fingers}

In this experiment, we have used 6 different combinations of two fingers: RIF-RMF, RIF-LMF, RIF-LIF, RMFLMF, RMF-LIF employing DRB classifier with different distances such as Euclidean, Cos, Correlation as well as Gabor filter bank and BSIF descriptor, and two rules, i.e., min and sum score level fusion. From the results of BSIFDRB and Gabor-DRB of two fingers presented in Table 5, 
Table 9 Comparison of the proposed multimodal system with the existing approaches for FKP

\begin{tabular}{lclllll}
\hline References & Year & Method & Performance & LIF-RIF & LIF-LMF & All finger \\
\hline Thapar et al. (2019) & 2019 & FKIMNet & EER (\%) & - & - & $2.03 \%$ \\
& & & Rank-1 (\%) & - & - & $94.02 \%$ \\
Zeinali et al. (2014) & 2014 & DFB +LDA & Rank-1 (\%) & $97.27 \%$ & $95.55 \%$ & $99.29 \%$ \\
Chlaoua et al. (2019) & 2019 & PCANet+SVM & EER (\%) & - & $0.404 \%$ & $0.00 \%$ \\
& & & Rank-1 (\%) & - & $97.84 \%$ & $100 \%$ \\
Jaswal et al. (2017b) & 2017 & DeepMatching & EER & - & - & $0.92 \%$ \\
Proposed system & 2020 & Gabor-DRB & EER (\%) & $2.44 \%$ & $3.01 \%$ & $1.82 \%$ \\
& & & Rank-1 (\%) & $98.13 \%$ & $97.32 \%$ & $99.24 \%$ \\
& & \multirow{2}{*}{ BISF-DRB } & EER (\%) & $2.33 \%$ & $3.08 \%$ & $\mathbf{0 . 1 9 \%}$ \\
& & & Rank-1 (\%) & $97.93 \%$ & $97.12 \%$ & $\mathbf{9 9 . 6 5 \%}$ \\
\hline
\end{tabular}

Our achieved results are indicated in bold we can observe that one modality trait using BSIF-DRB performs better than Gabor-DRB. Here, the proposed method using Gabor-DRB achieves higher accuracy on all combination of two fingers (RIF-RMF, RIF-LMF, RIFLIF, RMF-LMF, RMF-LIF) by applying min rules score level fusion. The accuracy ranges from 96.72 to $98.48 \%$ including all distances, and in comparison, to BSIF-DRB where the accuracy ranges from 95.05 to $97.37 \%$ including all distances. We also can see that the best fusion of two fingers according to the accuracy belongs to the first combination that is RIF-RMF for Gabor-DRB. The EER ranges from 2.17 to $2.93 \%$ including all distances for Gabor-DRB, and EER that ranges from 1.97 to $3.69 \%$ including all distances for BSIF-DRB, where we can see that the best EER achieved belongs to BSIF-DRB that equals to $1.99 \%$ but over all Gabor-DRB results were better.

The results of BSIF-DRB and Gabor-DRB of two fingers are presented in Table 6 using sum rule score level fusion. We can see that results over all are quite similar for both Gabor-DRB and BSIF-DRB including all distances, where the best accuracy belongs to RIF-RMF using Euclidean distance by accuracy equals to $98.03 \%$ for Gabor-DRB. While, for BSIF-DRB, the best accuracy is attained by RMF-LMF Euclidean distance, i.e., 98.32\% EER. We can observe more stability regarding Gabor$\mathrm{DRB}$, where it ranges from 1.79 to $2.63 \%$ for all distances and combinations. While, BSIF-DRB ranges from 1.47 to $2.83 \%$ for all distances and combinations. We can state that the best EER achieved belongs to BSIF-DRB by RMF-LM Fusing Euclidean distance.

Overall in comparison of min score level fusion results in Table 5 and sum rule score level fusion results in Table 6. For two fingers, it appears that min rule has achieved slightly better results in general; it was able to get an accuracy of $98.48 \%$ as best, while sum rule got $98.32 \%$.

\subsubsection{Experiment 2 and 3: three fingers and all fingers}

In this experiment, we have used 4 different combinations of three fingers: RIF-RMF-LMF, RIF-RMF-LIF, RIF-LMFLIF, RMF-LMF-LIF and all fingers combined employing DRB classifier with different distances such as Euclidean, Cos, Correlation as well as Gabor filter bank and BSIF descriptor and two rules, i.e., min and sum score level fusion.

In Table 7, we can see clearly the catching increase in the accuracy, regardless of the descriptor employed, using three fingers and all fingers fusion by applying min rule score level fusion. The best accuracy achieved by three fingers equals to $99.34 \%$ with RIF-RMF-LMF fingers fusion using GaborDRB and Euclidean distance, and $98.64 \%$ using BSIF-DRB and Euclidean distance by RMF-LMF-LIF three fingers. Whereas, the best EER equals to $1.17 \%$ with RIF-RMFLMF three finger fusion using Gabor-DRB and Euclidean distance, and $1.51 \%$ using BSIF_DRB and Euclidean distance by RIF-RMF-LMF fingers fusion. All in all, we can conclude that the Gabor-DRB is better than BSIF-DRB.

For all fingers fusion, we can observe in Table 7 that fusion using min score level fusion rule was able to attain the highest results, where the accuracy reached $99.65 \%$ using Gabor-DRB and Euclidean distance and EER equals to $0.91 \%$.

Table 8 shows the accuracy and EER achieved using three finger and all fingers fusion by applying sum rule. The best accuracy via three fingers equals to $98.89 \%$ with RIFRMF-LMF fingers fusion using Gabor-DRB and Euclidean distance, and $99.24 \%$ using BSIF-DRB and Euclidean distance by RMF-LMF-LIF three fingers. While, the best EER equals to $1.07 \%$ for RIF-RMF-LMF three finger fusion using Gabor-DRB and Euclidean distance, and 1.62\% using BSIF-DRB and Euclidean distance by RIF-RMF-LMF fingers fusion. Therefore, we can conclude that BSIF-DRB is better than Gabor-DRB using sum score level fusion rule. For all fingers fusion, we can observe in Table 8 that fusion 
Fig. 10 Curve ROC for all min score level fusion

Fig. 11 Curve ROC for all sum score level fusion
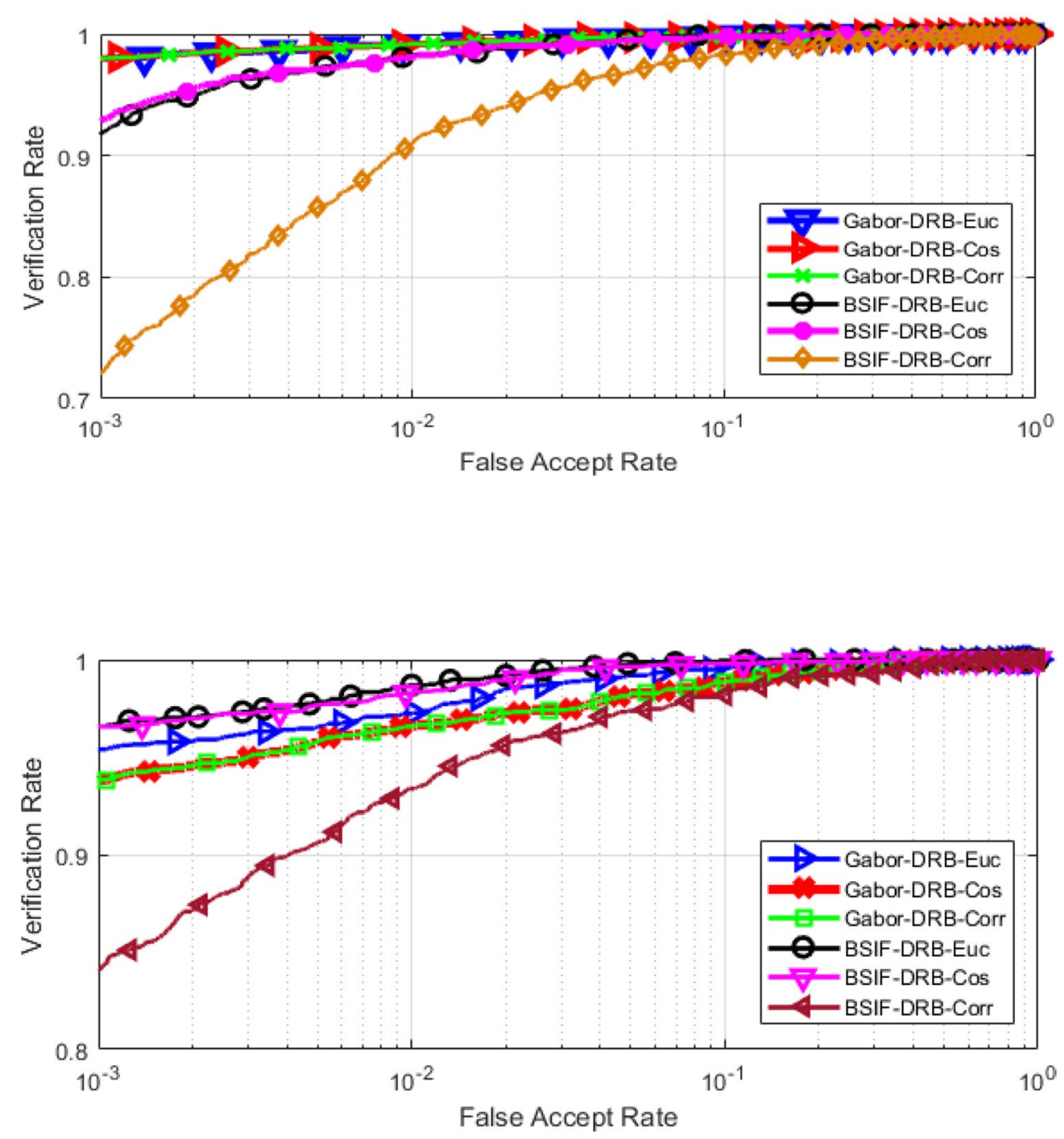

utilizing sum score level fusion rule has shown best results, where the accuracy reached $99.65 \%$ using BSIF_DRB and Euclidean distance and EER equals to $0.19 \%$.

By comparing the min score level fusion rule results in Table 7 with sum score level fusion rule results in Table 8 for three fingers, we can state that min rule has achieved better results in general where it was able to get an accuracy of $99.34 \%$ as the best, while sum rule reached $99.65 \%$ as highest accuracy. But overall min rule performed better. For all fingers, it was able to top all results obtained using two and three finger fusion such that it was able to get a $99.65 \%$ accuracy for both rules.

For further analysis of the results, we have obtained ROC curves to report comparison between the proposed method BSIF-DRB and Gabor-DRB with different distances utilizing FKP modality fusion and min rule in Fig. 10 and sum rule in Fig. 11. The results clearly show that the performance is better when the system uses the features of the Gabor filter bank with Euclidean distance.

Lastly, we can conclude from the previous experiment of single modality and the experiment using multi-trait (multimodal) that a multi-trait system has improved the performance of the proposed method, and as the number of modalities increases as the accuracy of the system.

\subsubsection{Comparison with prior studies on FKP}

The comparison of proposed multimodal system with existing systems in the literature is presented in Table 9. We can notice in Table 9 that the proposed system under verification mode attained lowest EER that is $0.19 \%$. compared to EER by systems in Jaswal et al. (2017b) and Thapar et al. (2019) for all fingers. In identification mode, for the modalities (LIF-RIF), (LIF-LMF) and all finger the proposed system 
achieved highest recognition rate (rank-1) compared to scheme designed in Zeinali et al. (2014). In addition, the presented system in this study achieved $99.65 \%$ of rank-1, which is better than FKIMNet proposed in Thapar et al. (2019). It can also be noted that work in Chlaoua et al. (2019) achieved the highest recognition rate $100 \%$ for all fingers, but if we observe for two fingers (e.g., LIF-RIF or LIFLMF) the proposed system reached a good performance.

\section{Conclusion}

This paper presents a new method for recognizing individuals based on their finger knuckle patterns (FKP). Specifically, the proposed method applied deep rule based (DRB) classifier on FKP. Together with DRB, two different feature extraction methods (i.e., Binarized Statistical Image Features and Gabor Filer bank) have be used. The results obtained on public PolyU dataset are quite impressive. Extensive empirical analyses were performed, in this study, using single modality and multi-traits/multimodal (two fingers and three fingers and all finger fusion) based on min and sum score level fusion rules. The obtained results demonstrated that DRB classifier is a powerful tool in person identification biometrics systems, especially in the case of multi-trait. The proposed method was able to attain accuracy comparable with existing schemes. Future work aims to realize a modified DRB classifier in computing similarities between features based on map diffusion. Also, the effectiveness of the proposed method under adversarial attacks will be studied.

\section{References}

Adeoye OS (2010) A survey of emerging biometric technologies. Int J Comput Appl 10:1-5

Akhtar Z, Fumera G, Marcialis GL, Roli F (2011a) Robustness evaluation of biometric systems under spoof attacks. In: Maino G, Foresti GL (eds) International conference on image analysis and processing. Springer, Berlin, pp 159-168

Akhtar Z, Biggio B, Fumera G, Marcialis GL (2011b) Robustness of multi-modal biometric systems under realistic spoof attacks against all traits. In: 2011 IEEE workshop on biometric measurements and systems for security and medical applications (BIOMS), pp 1-6

Angelov PP, Gu X (2017) Autonomous learning multi-model classifier of 0-Order (ALMMo-0)

Angelov PP, Gu X (2018) Deep rule-based classifier with human-level performance and characteristics. Inf Sci (Ny) 463-464:196-213

Angelov P, Soares E (2020) Towards explainable deep neural networks (xDNN). Neural Netw 130:185-194

Angelov P, Sperduti A (2016) Challenges in deep learning. ESANN, pp 489-494

Angelov P, Yager R (2012) A new type of simplified fuzzy rule-based system. Int J Gen Syst 41(2):163-185
Aoyama S, Ito K, Aoki T (2014) A finger-knuckle-print recognition algorithm using phase-based local block matching. Inf Sci (Ny) 268:53-64

Attia A, Chaa M, Akhtar Z, Chahir Y (2018) Finger kunckcle patterns based person recognition via bank of multi-scale binarized statistical texture features. Evol Syst 1-11

Bao R-J, Rong H-J, Angelov PP, Chen B, Wong PK (2018) Correntropy-based evolving fuzzy neural system. IEEE Trans Fuzzy Syst 26(3):1324-1338

Cappelli R, Ferrara M, Maltoni D (2010) Minutia cylinder-code: a new representation and matching technique for fingerprint recognition. IEEE Trans Pattern Anal Mach Intell 32(12):2128-2141

Chaa M, Boukezzoula N-E, Attia A (2017) Score-level fusion of twodimensional and three-dimensional palmprint for personal recognition systems. J Electron Imaging 26(1):13018

Chalabi NE, Attia A, Bouziane A (2020) Multimodal finger dorsal knuckle major and minor print recognition system based on PCANET deep learning. ICTACT J Image Video Process 10(3):2153-2158

Chlaoua R, Meraoumia A, Aiadi KE, Korichi M (2019) Deep learning for finger-knuckle-print identification system based on PCANet and SVM classifier. Evol Syst 10(2):261-272

El-Tarhouni W, Shaikh MK, Boubchir L, Bouridane (2014) A Multiscale shift local binary pattern based-descriptor for fingerknuckle-print recognition. In: Microelectronics (ICM), 2014 26th international conference on, pp 184-187

Ferrer MA, Travieso CM, Alonso JB (2005) Using hand knuckle texture for biometric identification. In: Proceedings 39th annual 2005 international carnahan conference on security technology, pp 74-78

Gu X, Angelov PP (2018) Semi-supervised deep rule-based approach for image classification. Appl Soft Comput 68:53-68

Gu X, Angelov PP, Zhang C, Atkinson PM (2018) A massively parallel deep rule-based ensemble classifier for remote sensing scenes. IEEE Geosci Remote Sens Lett 15(3):345-349

Hammouche R, Attia A, Akrouf S (2020) A novel system based on phase congruency and gabor-filter bank for finger knuckle pattern authentication. ICTACT J Image Video Process 10(3):2125-2131

Heidari H, Chalechale A (2020) A new biometric identity recognition system based on a combination of superior features in finger knuckle print images. Turkish J Electr Eng Comput Sci 28(1):238-252

Jaswal G, Kaul A, Nath R (2016) Knuckle print biometrics and fusion schemes-overview, challenges, and solutions. ACM Comput Surv 49(2):34

Jaswal G, Nigam A, Nath R (2017a) DeepKnuckle: revealing the human identity. Multimed Tools Appl 76(18):18955-18984

Jaswal G, Nigam A, Nath R (2017b) Finger knuckle image based personal authentication using DeepMatching. In: 2017 IEEE international conference on identity, security and behavior analysis (ISBA), pp 1-8

Kannala J, Rahtu E (2012a) Bsif: binarized statistical image features. In: 21 st international conference on pattern, and undefined. https ://ieeexplore.ieee.org

Kannala J, Rahtu E (2012b) Bsif: binarized statistical image features. In: Pattern recognition (ICPR), 2012 21st international conference on, pp 1363-1366

Liu C, Wechsler H (2002) Gabor feature based classification using the enhanced fisher linear discriminant model for face recognition. IEEE Trans Image Process 11(4):467-476

Malarvizhi N, Selvarani P, Raj P (2019) Adaptive fuzzy genetic algorithm for multi biometric authentication. Multimed Tools Appl $1-14$ 
Muthukumar A, Kavipriya A (2019) A biometric system based on gabor feature extraction with SVM classifier for finger-knuckleprint. Pattern Recognit Lett 125:150-156

PolyU (2010) The Hong Kong polytechnic university (PolyU) FingerKnuckle-Print Database. [Online]. https://www.comp.polyu.edu. hk/biometrics/FKP.html

Qian J, Yang J, Tai Y, Zheng H (2016) Exploring deep gradient information for biometric image feature representation. Neurocomputing 213:162-171

Sargano AB, Wang X, Angelov P, Habib Z (2017) Human action recognition using transfer learning with deep representations. In: 2017 International joint conference on neural networks (IJCNN), pp 463-469

Shariatmadar ZS, Faez K (2013) Finger-knuckle-print recognition via encoding local-binary-pattern. J Circuits Syst Comput 22(6): 1350050

Shen L, Bai L (2006) A review on gabor wavelets for face recognition. Pattern Anal Appl 9(2-3):273-292

Singh S, Kant C (2019) FKP and Iris based multimodal biometric system using PCA with NFNN. Available SSRN 3358136

Soltanpour S, Boufama B, Wu QMJ (2017) A survey of local feature methods for 3D face recognition. Pattern Recognit 72:391-406

Thapar D, Jaswal G, Nigam A (2019) FKIMNet: a finger dorsal image matching network comparing component (major, minor and nail) matching with holistic (finger dorsal) matching. arXiv Prepr. arXiv1904.01289
Wang R, Han C, Wu Y, Guo T (2014) Fingerprint classification based on depth neural network. arXiv Prepr. arXiv1409.5188

Woodard DL, Flynn PJ (2005) Finger surface as a biometric identifier. Comput Vis Image Underst 100(3):357-384

Zeinali B, Ayatollahi A, Kakooei M (2014) A novel method of applying directional filter bank (DFB) for finger-knuckle-print (FKP) recognition. In: Electrical engineering (ICEE), 2014 22nd Iranian conference on, pp 500-504

Zhai Y et al. (2018) A novel finger-knuckle-print recognition based on batch-normalized CNN. In: Chinese conference on biometric recognition, pp 11-21

Zhang L, Zhang L, Zhang D, Zhu H (2011) Ensemble of local and global information for finger-knuckle-print recognition. Pattern Recognit 44(9):1990-1998

Zhang L, Zhang L, Zhang D, Guo Z (2012) Phase congruency induced local features for finger-knuckle-print recognition. Pattern Recognit 45(7):2522-2531

Zhang D, Lu G, Zhang L (2018) Finger-knuckle-print verification. In: Zhang D, Lu G, Zhang L (eds) Advanced biometrics. Springer, Cham, pp 85-109

Publisher's Note Springer Nature remains neutral with regard to jurisdictional claims in published maps and institutional affiliations 